\title{
Docencia en medicina de urgencias y emergencias
}

\section{Teaching in emergency medicine}

\author{
F. Ayuso' ${ }^{1}$, R. Nogué2, B. Coll Vinent ${ }^{3}$, B. Fernández Esáin ${ }^{4}$, Ò. Miró ${ }^{5}$
}

\section{RESUMEN}

El manejo adecuado de los pacientes en los servicios de urgencias y emergencias puede llegar a ser lo suficientemente complejo como para requerir una sólida formación que debiera estar sustentada en una especialidad médica, como ocurre en la mayoría de países de nuestro entorno.

En la actualidad, en España no existe una formación reglada y homogénea en medicina de urgencias y emergencias (MUE) ni durante el período de formación de pregrado (en forma de asignatura obligatoria de forma universal en las facultades de medicina) ni durante el período de posgrado (en forma de especialidad médica). En este sentido, actualmente se está pendiente de la aprobación definitiva de esta especialidad en MUE en el marco de una reforma del programa de residencia que evolucionará hacia un programa formativo troncal de especialidades. A la espera de ello, la realidad es que en España los profesionales que desarrollan su labor en este ámbito asistencial poseen una formación heterogénea. Fruto de todo este vacío y de las necesidades formativas de estos profesionales, se ha desarrollado con los años un amplio abanico de propuestas formativas específicas para optimizar las habilidades y destrezas de los profesionales que prestan la primera asistencia urgente a los pacientes.

Una nueva generación de cursos se ha puesto en marcha utilizando las nuevas metodologías didácticas de formación, en las que se incorporan herramientas didácticas de e-learning y simulación robótica.

Palabras clave. Docencia. Medicina de urgencias. Emergencias.

\begin{abstract}
The appropriate care of patients in emergency services can reach a level of complexity as to make a sound training necessary, which should be based on a medical speciality, as happens in the majority of the countries in our context.

In Spain at present there is no regulated and homogeneous training in urgency and emergency medicine (UEM), either during the period of undergraduate training (in the form of a universally compulsory subject in the faculties of medicine) or during the postgraduate period (in the form of a medical speciality). In this respect, the definitive approval of the speciality in UEM is currently pending within the framework of a reform of the residence program that will evolve towards a core training program of specialities. Until thus occurs, the reality in Spain is that professionals who work in this care setting possess a heterogeneous training. As a result of this vacuum and the training needs of these professionals, a wide range of specific training proposals has been developed over the years in order to optimise the skills and abilities of the professionals who provide initial emergency care to the patient.
\end{abstract}

A new generation of courses has been set underway using the new didactic methodologies of training, into which didactic tools of e-learning and robotic simulation have been incorporated.

Key words. Teaching. Emergency medicine. Emergencies.
1. Empresa Pública de Emergencias Sanitarias. EPES 061. Córdoba.

2. Servicio de Urgencias. Hospital Arnau de Vilanova. Lleida.

3. Área de Urgencias. Hospital Clinic. Barcelona. 4. Servicio de Urgencias. Hospital Navarra. Pamplona.

5. Servicio de Urgencias. Hospital Clinic.

Barcelona. Director Revista Emergencias.

\section{Correspondencia}

Fernando Ayuso Baptista

Empresa Pública de Emergencias Sanitarias

EPES 061. Córdoba

E-mail: fayuso@co.epes.es 


\section{INTRODUCCIÓN}

El manejo adecuado de los pacientes en los servicios de urgencias y emergencias puede llegar a ser lo suficientemente complejo como para requerir una sólida formación universitaria de pregrado, que debiera estar posteriormente sustentada en una formación especializada a través de una residencia, que es lo que ocurre en muchos de los países de nuestro entorno. Tras esta formación inicial, es preciso un entrenamiento periódico en situaciones clínicas y en escenarios simulados que doten a los equipos intervinientes de las habilidades necesarias para poder desarrollar una labor asistencial eficiente dentro de unos estándares de calidad mínimamente exigidos y que aseguren una formación continuada de calidad al especialista ${ }^{1}$.

En la actualidad, ante la ausencia de especialidad en medicina de urgencias y emergencias (MUE) en nuestro país, nos encontramos con que los profesionales que desarrollan su labor en ese ámbito asistencial poseen una formación heterogénea. Es necesario que los profesionales puedan acceder a acciones formativas de diferente complejidad, aunque todas ellas deberían tener como objetivo entrenarles de una forma integral que permita ofrecer una asistencia coordinada entre médicos, enfermeros y técnicos en emergencias. La complejidad del manejo de estos pacientes viene dada, entre otras cosas, por la concurrencia en urgencias de la totalidad de los procesos asistenciales, además del hecho de tratarse de situaciones que generan gran ansiedad por el escenario en el que aparecen. Se trata, además, de situaciones altamente impactantes, en las cuales deben tomarse decisiones rápidas, lo que obliga a ajustarse a procesos metodológicos claramente establecidos y reglados, que deben automatizarse $^{1}$. Para ello es imprescindible, mediante la formación específica en MUE, optimizar las habilidades y destrezas de los profesionales que prestan la primera asistencia a los pacientes con el fin de que adquieran una sistemática activa de valoración que estratifiquen el riesgo individual inicial de cada paciente y que ejecuten las acciones terapéuticas precisas que conlleven un beneficio inmediato del paciente ${ }^{2}$.

\section{DESARROLLO HISTÓRICO DE LA FORMACIÓN EN MEDICINA DE URGENCIAS Y EMERGENCIAS EN ESPAÑA}

La metodología docente de los programas formativos destinados a los profesionales de urgencias y emergencias debe tener como objetivo proporcionar al alumno una herramienta contrastada y segura para la evaluación y tratamiento inicial del paciente, así como las habilidades técnicas necesarias para valorar al paciente de forma precisa y rápida, instaurar un soporte vital, establecer en cada fase del tratamiento las medidas óptimas de atención e identificar con prontitud el centro útil para el manejo adecuado y seguro del paciente $e^{2,3}$.

Con esta finalidad, en los años 80, los programas de formación en politraumatrismos (ATLS, Advanced Trauma Life Support) se diseñaron combinando sesiones teóricas con talleres prácticos y utilizaban metodología demostrativa que incluía sesiones de discusión, así como prácticas en laboratorio de cirugía animal. Posteriormente, se incorporó la evaluación teórica antes y después del curso así como la evaluación práctica utilizando pacientes simu$\operatorname{lados}^{2,3}$.

Con posterioridad, durante los años 90 se desarrollaron una serie de cursos, másteres, especializaciones universitarias (Lleida, Córdoba, Sevilla, Madrid, La Coruña, Santander, Barcelona, etc.) en un intento de paliar el déficit formativo existente y, muchos de ellos, se siguen impartiendo a día de hoy. Por su parte, durante esos años la Sociedad Española de Medicina de Urgencias y Emergencias (SEMES) promovió una acreditación tanto para médicos como para enfermeros y técnicos de transporte sanitario (certificados de medicina, enfermería y técnicos de transporte sanitario de Emergencias). Este programa, actualmente vigente, tiene por objetivo certificar la formación necesaria para la práctica de la atención urgente con la perspectiva de una 
futura oficialización, la cual ya se ha producido en el caso de los técnicos. Para la obtención de dicho certificado es preciso la superación de una prueba anual y única a nivel nacional.

Durante estos últimos años, se ha puesto en marcha una nueva generación de cursos que utilizan las nuevas metodologías didácticas de formación, entre ellos las acciones formativas en las que se incorporan herramientas didácticas de e-learning. Los cursos desarrollados con simulación robótica con el empleo de simuladores, herramientas didácticas de dramatización, escenografía veraz y posterior análisis de las asistencias simuladas, tras ser grabadas en vídeo, han supuesto un salto cualitativo importante en la metodología didáctica de la $\mathrm{MUE}^{2}$.

\section{SITUACIÓN ACTUAL DE LA ENSEÑANZA DE LA MEDICINA DE URGENCIAS Y EMERGENCIAS EN LAS UNIVERSIDADES ESPAÑOLAS}

En nuestro país, la enseñanza de la MUE como disciplina específica no está regulada durante la licenciatura ni después de finalizarla. Si analizamos cuál ha sido la evolución de la enseñanza de pregrado, observaremos que durante los últimos años, y de una forma creciente, las facultades de medicina españolas han introducido en su currículum la asignatura de MUE. Así, en la actualidad, esta disciplina se enseña como asignatura en 22 de las 33 facultades de medicina de las universidades españolas (67\%), y en otras 3 de ellas está programado impartirla en los próximos años ${ }^{4}$. En 6 de estas facultades se imparte más de una asignatura de medicina de urgencias y, entre ellas, difieren fundamentalmente en el temario o en la unidad docente en la que son impartidas. Esto hace un total de 29 asignaturas (Tabla 1) y en todos los casos éstas se imparten en el segundo ciclo de la carrera; la mayoría son optativas o de libre elección (79\%) y su duración suele ser cuatrimestral $(93 \%)^{3}$. La mediana de créditos ofrecidos, si se supera la asignatura, es de 5 (mínimo 2, máximo 9) y en la mayoría de los casos es de 4,5. El número de créditos teóricos es similar al número de créditos prácticos. El programa es muy variable tanto en extensión como en contenidos. El número de temas teóricos varía de 4 a 27 y el temario es muy heterogéneo, con un enfoque generalmente sindrómico (83\%). El programa práctico varía desde 4 horas de seminarios de habilidades clínicas a 48 horas de prácticas clínicas obligatorias en servicios de urgencias. La reanimación cardiopulmonar es el tema más prevalente en los programas disponibles, tanto teóricos como prácticos, aunque tampoco se llega a dar en el 100\% de las asignaturas.

En 2002, la SEMES hizo una propuesta para homogeneizar la enseñanza de la MUE durante la licenciatura de Medicina y Cirugía ${ }^{5}$. En ella destacaba la obligatoriedad de la asignatura, los créditos ofrecidos (3 teóricos, 4 prácticos), la necesidad de impartir la asignatura en segundo ciclo y de realizar unas prácticas asistenciales durante un mínimo de un mes, así como la existencia de un programa teórico y práctico detallado. Muy recientemente se han publicado unas bases de consenso internacional por parte de la IFEM (Internacional Federation of Emergency Medicine) acerca de los contenidos mínimos que debieran impartirse durante el período de pregrado a los estudiantes de medicina de todo el mundo ${ }^{6}$.

Sin embargo, la realidad es que, en el momento actual, España se encuentra muy lejos de estas propuestas. Cada facultad tiene su propio criterio, ignorando de una u otra manera estas recomendaciones. Sin duda es necesaria una tarea de difusión de las recomendaciones, pero las enormes diferencias existentes entre éstas y algunos programas sugieren que, probablemente, sea necesario consensuarlas y actualizarlas. Presumiblemente, el reconocimiento y la instauración de la MUE como especialidad médica acarreen una homogeneidad y una normalización en la enseñanza de esta materia durante la licenciatura, a la vez que probablemente favorecerá la creación de cátedras específicas para esta disciplina, prácticamente inexistentes en la actualidad. 
Tabla 1. Características generales de la asignatura de urgencias en las distintas facultades de medicina de España.

\begin{tabular}{|c|c|c|c|c|c|}
\hline Facultad & Núm. & Nombre & $\begin{array}{c}\text { Tipo de } \\
\text { asignatura }\end{array}$ & Duración & Créditos (T/P)* \\
\hline \multirow[t]{2}{*}{ Cádiz } & \multirow[t]{2}{*}{2} & $\begin{array}{l}\text { Medicina de emergencias y } \\
\text { catástrofes }\end{array}$ & Optativa & Cuatrimestral & $5(3 / 2)$ \\
\hline & & Urgencias y medicina intensiva & Optativa & Cuatrimestral & $5(3,5 / 1,5)$ \\
\hline Córdoba & 1 & $\begin{array}{l}\text { Medicina de urgencias y } \\
\text { emergencias }\end{array}$ & Libre elección & Cuatrimestral & $4,5(2 / 2,5)$ \\
\hline \multirow[t]{2}{*}{ Granada } & \multirow[t]{2}{*}{2} & Urgencias médico-quirúrgicas & Optativa & Cuatrimestral & $5(2,5 / 2,5)$ \\
\hline & & Introducción a las urgencias & Libre elección & Cuatrimestral & $2(0 / 2)$ \\
\hline Màlaga & 1 & $\begin{array}{l}\text { Medicina intensiva y urgencias } \\
\text { médicas }\end{array}$ & Obligatoria & Cuatrimestral & $4,5(2,5 / 2)$ \\
\hline \multirow[t]{2}{*}{ Sevilla } & \multirow[t]{2}{*}{2} & Medicina y cirugía de urgencias & Obligatoria & Cuatrimestral & $4,5(3 / 1,5)$ \\
\hline & & $\begin{array}{l}\text { Actuación inicial en emergencias } \\
\text { sanitarias extrahospitalarias }\end{array}$ & Libre elección & Cuatrimestral & $3(1,5 / 1,5)$ \\
\hline Zaragoza & 1 & $\begin{array}{l}\text { Urgencias, emergencias y } \\
\text { medicina intensiva }\end{array}$ & Optativa & Cuatrimestral & $6(3 / 3)$ \\
\hline \multirow[t]{2}{*}{ Oviedo } & \multirow[t]{2}{*}{2} & Emergencias quirúrgicas & Optativa & Cuatrimestral & $4,5(2,5 / 2)$ \\
\hline & & Urgencias médicas & Optativa & Cuatrimestral & $5(2,5 / 2,5)$ \\
\hline Las Palmas & 1 & Medicina de urgencias & Obligatoria & Anual & $5(2,5 / 2,5)$ \\
\hline Cantabria & 1 & Medicina de urgencia & Obligatoria & Cuatrimestral & $7(3 / 4)$ \\
\hline Salamanca & 1 & Medicina de urgencias & Optativa & Cuatrimestral & $4(2 / 2)$ \\
\hline Valladolid & 1 & $\begin{array}{l}\text { Urgencias y emergencias en } \\
\text { medicina }\end{array}$ & Optativa & Cuatrimestral & $4,5(2 / 2,5)$ \\
\hline \multirow[t]{2}{*}{ Barcelona } & \multirow[t]{2}{*}{2} & $\begin{array}{l}\text { Puntos clave en la atención } \\
\text { urgente a un paciente }\end{array}$ & Optativa & Cuatrimestral & $6(5 / 1)$ \\
\hline & & Prácticas de urgencias & Libre elección & Cuatrimestral & $6(0 / 6)$ \\
\hline \multirow{4}{*}{$\begin{array}{l}\text { Autónoma Barcelona } \\
\text { UD Valle Hebrn } \\
\text { UD Can Ruti } \\
\text { UD Sant Pau }\end{array}$} & & & & & \\
\hline & 1 & Medicina de urgencia & Optativa & Cuatrimestral & 4,5 \\
\hline & 1 & Medicina de urgencia & Optativa & Cuatrimestral & $4,5(1,5 / 3)$ \\
\hline & 1 & Medicina de urgencia & Optativa & Cuatrimestral & $4,5(1,5 / 3)$ \\
\hline Lleida & 1 & Urgencias y medicina intensiva & Optativa & Cuatrimestral & 4 \\
\hline $\begin{array}{l}\text { Rovira i Virgili de Reus } \\
\text { (Tarragona) }\end{array}$ & 1 & Medicina de urgencia & Optativa & Cuatrimestral & $7,5(3 / 4,5)$ \\
\hline Extremadura & 1 & $\begin{array}{l}\text { Anestesiología, reanimación y } \\
\text { urgencias clínicas }\end{array}$ & Obligatoria & Anual & $9(4 / 5)$ \\
\hline Santiago de Compostela & 1 & Medicina y urgencia clínica & Optativa & Cuatrimestral & $4,5(3 / 1,5)$ \\
\hline Complutense Madrid & 1 & Urgencias en medicina & Libre elección & Cuatrimestral & $9(3 / 6)$ \\
\hline Autónoma Madrid & 1 & $\begin{array}{l}\text { Reanimación cardiopulmonar y } \\
\text { actuaciones urgentes básicas }\end{array}$ & Optativa & Cuatrimestral & 4,5 \\
\hline País Vasco & 1 & Medicina de urgencias & Obligatoria & Cuatrimestral & $5(2 / 3)$ \\
\hline $\begin{array}{l}\text { Miguel Hernández de } \\
\text { Elche (Alicante) }\end{array}$ & 1 & Medicina de urgencias & Optativa & Cuatrimestral & $5(2 / 3)$ \\
\hline Valencia & 1 & Urgències mediques & Optativa & Cuatrimestral & $6(0,5 / 5,5)$ \\
\hline
\end{tabular}

*T/P: teóricos/prácticos, UD: unidad docente 


\section{LA FORMACIÓN ESPECIALIZADA EN MEDICINA DE URGENCIAS EN EUROPA}

Antes de abordar la situación en España, resulta fundamental conocer y comparar la realidad existente en los países de nuestro entorno, especialmente en los del espacio europeo de educación superior, ya que la finalidad última de este espacio es diseñar currículos formativos similares que favorezcan el reconocimiento y la convergencia de titulaciones. Ello ha de permitir realizar una mejor planificación de futuro en cuanto al desarrollo de la especialidad de MUE en España, por cuanto se pueden copiar los aciertos de dichos sistemas e intentar corregir los errores cometidos. Actualmente, existen bastantes diferencias entre los diversos países que son debidas a condicionantes históricos, formativos, de organización del propio sistema sanitario y de necesidad de dar respuesta a los nuevos retos en materia de atención sanitaria. A continuación se presenta un análisis sucinto de la situación a finales de 2009 .

Según la directiva 2005/36/EC ${ }^{7}$ de la Comisión Europea, la especialidad de MUE existe en 7 países (Reino Unido e Irlanda -en éstos desde hace mas de 20 años-, República Checa, Irlanda, Hungría, Malta, Polonia y Eslovaquia -éstos últimos de aprobación más reciente-). Con posterioridad a esta directiva, la especialidad ha sido aprobada en Rumanía y Eslovenia. En promedio, la duración de la residencia es de unos 5 años $^{8-10}$. Por otro lado, fuera de Europa, la especialidad también está plenamente reconocida en Estados Unidos, Canadá, Filipinas, Australia y México. En todos estos países existen unos planos formativos perfectamente definidos y establecidos cuyo cumplimiento es obligatorio por parte de los centros y de los especialistas en formación para que se pueda obtener el título de especialista.

En otros países, la formación está asegurada con complementos formativos de acceso selectivo al acabar alguna de las especialidades de base. En general, estos diplomas son condición necesaria para ini- ciarse en el ámbito asistencial, progresar profesionalmente o dirigir las estructuras de atención urgente. Es el caso de Francia, la especialidad de MUE existe desde octubre de 2004 a través de los diplomas de estudios especializados complementarios (DESC), a los que se accede al finalizar medicina de familia, anestesia, cardiología, medicina interna, traumatología o pediatría. Constituye una formación complementaria de dos años adicionales con rango de especialidad y son comunes las convalidaciones según la especialidad de origen. En ese país, el modelo descrito coexiste con las Capacite de MUE que regulaban la formación desde el año 1988. La titulación es imprescindible para presentarse al concurso nacional de adjudicación de puestos y tiene la denominación de Medicina Polivalente de Urgencias. Por su parte, en Bélgica existen desde 1993 dos niveles de formación: el título profesional en MUE con formación de dos años a tiempo completo en un servicio de urgencias integral (hospitalario y extrahospitalario) y un "BREVET en medicina aguda" dirigido a médicos generales y realizada a tiempo parcial. En Alemania ${ }^{11}$ e Italia, la MUE es una subespecialidad con formación a tiempo parcial de 2 y 3 años de duración, respectivamente. En Portugal existe un diploma universitario de MUE, intra y extrahospitalario, validado por el Instituto Nacional de Urgencias Médicas (INEM) de dos años de duración. En los países nórdicos continentales no existe la especialidad de MUE ${ }^{12}$ : son habitualmente los médicos de los diferentes servicios los que intervienen según la patología presentada. La frecuentación de dichos países es comparativamente baja en relación a nuestra casuística (alrededor de 100 consultas/1.000 habitantes en Finlandia y Noruega y 200/1.000 en Suecia), probablemente porque las derivaciones al hospital deben ser previamente filtradas. En Suecia existen iniciativas para la creación de la especialidad, la cual ya está presente en Islandia desde 2006. Finalmente, con respecto a los países del área mediterránea, la MUE es una especialidad en Marruecos (desde 2006), Turquía e Israel y en Argelia existe una Capacite similar a la francesa. 


\section{PROYECTO DE FORMACIÓN DE LOS NUEVOS ESPECIALISTAS EN MEDICINA DE URGENCIAS Y EMERGENCIAS EN ESPAÑA}

Como se comentaba anteriormente, en España no existe a día de hoy una formación de posgrado reglada en MUE. Técnicamente, el acceso a la especialidad de MUE sería posible a través de un sistema de área de capacitación específica ${ }^{13}$ (ACE, arts. 24 y 25 de la LOPS), que consiste en la formación adicional desde otras especialidades en la cual se otorga un diploma, con 5 años de trabajo y formación y evaluación del profesional. Este sistema tiene ciertas ventajas (como la flexibilidad, permeabilidad y facilidad de implantarse a corto plazo), pero resultan mayores los inconvenientes que plantea (como son la diversidad de perfiles profesionales, formación compleja, larga y sin uniformidad). La alternativa a este tipo de formación lo constituye el desarrollo de una especialidad reglada, que es la vía que apoya la Federación Europea de Sociedades de Medicina de Urgencias y Emergencias (EuSEM) y, obviamente, la SEMES ${ }^{14}$. Las diferentes sociedades, y de acuerdo con la Directiva Europea ${ }^{7}$, contemplan un período formativo de al menos 5 años a través de una formación post-grado, basándose en un cuerpo doctrinal específico. Recientemente, la EuSEM ha hecho público un documento de consenso en el que se recogen todos los requerimientos formativos que deben cumplir los residentes de MUE en Europa, el cual es de obligada consulta para aquellos países que quieran desarrollar la especialidad ${ }^{14}$.

En España, el desarrollo de esta especialidad a través de esta vía debe concretarse en una formación de posgrado a través de una residencia estructurada del tipo MIR. En este sentido, y con objeto de corregir la situación actual de superespecialización (ventajas: mejoría de la calidad asistencial, avance rápido de conocimientos y de técnicas; inconvenientes: asistencia compartimentada, necesidad de varios especialistas para atender un paciente) y mejorar la formación básica común de los especialistas, la Ley de Ordenación de las Profesiones Sanitarias (LOPS) ha replanteado los progra- mas formativos introduciendo el concepto de troncalidad. Así, se ha diseñado un programa formativo troncal de especialidades ${ }^{13}$ (art. 19.2 y 3 y art. 21.2 de la LOPS) donde varias especialidades comparten un programa formativo común de al menos 2 años y que se completa con una formación de hasta 3 años adicionales para la obtención del título de especialista. Esto ha de proporcionar mayor homogeneidad en la formación y mayores posibilidades de planificación que el sistema vigente en la actualidad. La agrupación de determinadas especialidades en un tronco común, compartiendo tramos comunes en su formación, supone un paso intermedio en la formación previo a la especialización definitiva. La troncalidad cobra un mayor sentido, si cabe, al permitir realizar la elección definitiva de la especialidad de forma más madura y meditada ${ }^{15}$. La troncalidad no ancla a la persona a una especialidad sino a un abanico de especialidades dentro del tronco común, con posibilidad de elección de la especialidad al inicio (similar al sistema actual, lo cual supone una planificación más fácil) o al final de la formación troncal (decisión más meditada para el profesional pero de mayor complejidad para la planificación del sistema). Asímismo, permite la movilidad racional entre profesionales del mismo tronco (art. 23 LOPS), lo cual es una garantía de asistencia sanitaria en determinados ámbitos geográficos.

Dentro de este proyecto de troncali$\operatorname{dad}^{15,16}$, existe un borrador para que se incluyan en un mismo tronco las especialidades de MUE, medicina familiar y comunitaria y medicina interna. La formación troncal común se desarrollará a lo largo de 2 años (art. 19 LOPS) y las competencias durante este periodo se adquirirían mediante rotaciones clínicas planificadas (en base a objetivos cognitivos y destrezas a conseguir) complementadas con cursos, seminarios, etc. (Tabla 2). En global, se reconocen tres tipos diferentes de competencias (art. 21 de la LOPS):

1. Competencias genéricas, que se desarrollarán a lo largo de 2 años y que incluyen rotaciones clínicas complementadas con cursos de estadística básica, epidemiología y gestión sanitaria, etc. 
Tabla 2. Rotación en los dos primeros años de residencia de los futuros MIR de Urgencias y Emergencias en España.

\begin{tabular}{|l|l|c|l|}
\hline Año de residencia & \multicolumn{1}{|c|}{ Rotación } & Duración meses & \multicolumn{1}{|c|}{ Guardias/mes } \\
\hline \multirow{4}{*}{$\mathbf{R 1}$} & Urgencias & 3 & URG HOSP 3 \\
MED INTERNA 1 \\
PAC-SEM 1
\end{tabular}

2. Competencias transversales, en las que deben incluirse las rotaciones por cardiología (2 meses), endocrinología (1 mes), diagnóstico por imagen (2 meses), atención primaria (3 meses), medicina interna (5 meses), digestivo (2 meses), neumología (2 meses), neurología (2 meses) y urgencias y emergencias (3 meses).Competencias específicas, que deben definirse por cada especialidad basadas en el cuerpo doctrinal propio. Los mecanismos por los cuales deben adquirirse estas competencias y habilidades son el autoaprendizaje, el aprendizaje del campo (auditoría de historias, intervenciones autorizadas, opiniones de profesionales del equipo o de otro nivel, vídeo-grabaciones y análisis posterior), las sesiones (clínicas, bibliográficas), el trabajo grupal (mejora de calidad, proyectos de investigación) y los talleres (pacientes simulados, maniquíes de simulación, centros específicos de entrenamiento (CEMV)).

En todo este proyecto quedan pendientes de reflexión varios aspectos ${ }^{13,15,17}$, como el hecho de qué ocurre cuando un médico se plantea la obtención de una segunda o tercera especialidad dentro de la formación troncal (art. 21 LOPS) o cuando, estando en el periodo específico de formación, decide cambiar de especialidad a otra de la misma o de distinta troncalidad. En el primer caso, podría accederse directamente a la formación específica mediante la evaluación del aspirante en la nueva especialidad, aunque quedaría por definir la duración y el contenido de la formación en función de los resultados obtenidos. En el caso de que el aspirante opte a otra especialidad de diferente troncalidad será obligada la formación común y específica de la misma ${ }^{16}$.

\section{LA FORMACIÓN DE POSTGRADO ACTUAL EN ESPAÑA}

La ausencia de asignatura de urgencias en los años anteriores, la ausencia de especialidad de urgencias y la enorme demanda de médicos en los servicios de urgencias ha favorecido la proliferación de programas extrauniversitarios, cursos de postgrado y másteres universitarios, en los cuales los médicos y demás profesionales que desean trabajar en urgencias buscan soporte ${ }^{18,19}$. Existen centenares de cursos de este tipo, así como una enorme variedad de los mismos, tanto respecto al temario impartido como al público al que va destinado o al 
método docente. Tanto los másteres universitarios como los cursos de iniciativa privada exigen una financiación particular importante (entre 3000 a $5.000 €$ ).

Los créditos ofrecidos varían en función de las horas, del método docente (presencial, on-line o a distancia) y del tipo de programa (postgrado, máster). Los másteres suelen ofrecer entre 60 y 120 créditos ECTS (European Credit Transfer System) ${ }^{20}$. Los requisitos para matricularse suelen ser mínimos (habitualmente el interés por la materia, el abono del importe y el estar en posesión de cuando menos una diplomatura) y los destinatarios suelen ser de distintas disciplinas, fundamentalmente de enfermería, medicina y técnicos. Es habitual, incluso en másteres universitarios oficiales, que los destinatarios sean dos o tres de estos colectivos simultáneamente. Por el contrario, los cursos destinados únicamente a médicos son pocos.

El temario también es muy variable. Esencialmente existen dos grandes grupos de temas: las de urgencias y emergencias en general, a menudo entrelazados con los cuidados críticos, y los cursos de emergencias y grandes catástrofes, en los que los sistemas de triaje, la reanimación cardiopulmonar y los politraumatismos son los temas principales, cuando no los únicos. Por tanto, aunque actualmente todos estos cursos son un buen sucedáneo para la formación en urgencias y a menudo la única forma disponible de asegurar una formación en esta disciplina, falta un gran trabajo de homogenización y acreditación de los mismos. Cabe esperar que, aunque la aprobación de la especialidad de MUE se produzca en un breve plazo, esta formación aún sea largamente demandada por aquellos profesionales procedentes de diversos ámbitos que a día de hoy ya ejercen la MUE.

\section{METODOLOGÍA DOCENTE ACTUAL EN URGENCIAS Y EMERGENCIAS}

Finalmente, vamos a hacer una mención especial a una nueva generación de cursos que se han puesto en marcha, los cuales utilizan las nuevas metodologías didácticas de formación. Entre ellos, se encuentran las acciones formativas diseñadas en dos módulos consecutivos: un módulo inicial en el que se utilizan las herramientas didácticas de e-learning a través de una plataforma de teleformación, seguido de un segundo módulo presencial desarrollado en talleres prácticos. En estas innovadoras acciones, el periodo de estudio previo y las clases magistrales presenciales se han sustituido por un periodo de autoestudio tutelado a través de una plataforma de teleformación, apoyado por material didáctico interactivo y multimedia. Este primer módulo permite que el alumno adquiera las competencias de conocimiento con la ayuda de este material didáctico diseñado ex profeso, herramientas de comunicación y debate (foros, chat, mensajería), actividades de teleformación (casos clínicos, cuestionarios, etc.) y recursos virtuales de consulta (biblioteca virtual, enlaces de interés, etc.), además del apoyo continuado de docentes expertos con formación específica en formación a distancia a través de este tipo de plataforma, que orientan al alumno en su proceso de aprendizaje, resuelven sus dudas y le motivan ${ }^{2,3}$. Sin duda, los avances de los últimos años en telemedicina, tanto aplicados al campo de la asistencia como la docencia, han permitido y potenciado este tipo de formación ${ }^{21}$.

En estas acciones formativas, después de este primer módulo de teleformación, se lleva a cabo un segundo módulo presencial, dirigido fundamentalmente a la adquisición de competencias de habilidades técnicas, desarrollado en talleres prácticos (Fig. 1) y que utiliza una metodología demostrativa y de simulación que propicia la continua participación de los discentes ${ }^{2}$. La simulación médica consiste, fundamentalmente, en crear un sistema o situación que reproduzca la realidad, con el objetivo de practicar o evaluar y, por ello, se puede incorporar al proceso de aprendizaje de situaciones complejas en el ámbito del entrenamiento sanitario ${ }^{2,3}$. Estos simuladores se caracterizan por sus avanzadas características anatómicas y fisiológicas (cardiovasculares y respiratorias, fundamentalmente) y por la posibilidad de realización 
de numerosas técnicas de entrenamiento (intubación, control de la vía aérea con dispositivos alternativos, cricotirotomía, drenaje neumotórax, canalización venosa, desfibrilación, etc.) (Fig. 2) 2,22. En la metodología de simulación robótica no sólo es primordial el realismo externo del propio maniquí, sino que además es imprescindi-

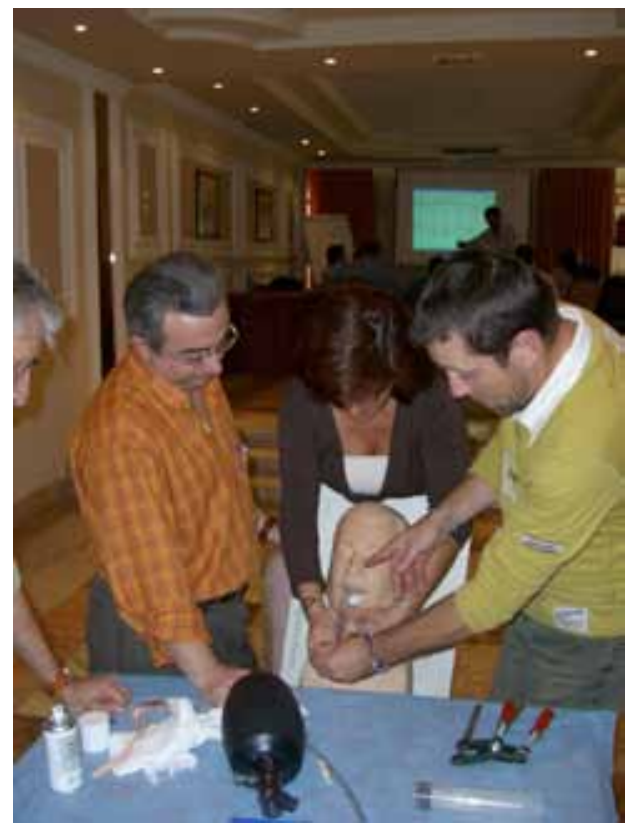

Figura 1. Programa de entrenamiento en situaciones de emergencias respiratorias en que se adiestra a los médicos en el manejo de la vía aérea en escenarios de dificultad.

En la simulación, como es obvio, pueden cometerse errores sin daño a pacientes, y de estos errores los alumnos pueden extraer conclusiones que ayuden a mejorar ble cuidar los elementos escenográficos y la idoneidad del material sanitario para crear un entorno de trabajo realista que potencie el conjunto de la simulación y facilite la consecución de los objetivos (salas de críticos, consultas e incluso escenarios prehospitalarios como domicilio o una vía urbana) (Fig. 3) $)^{2,3}$.

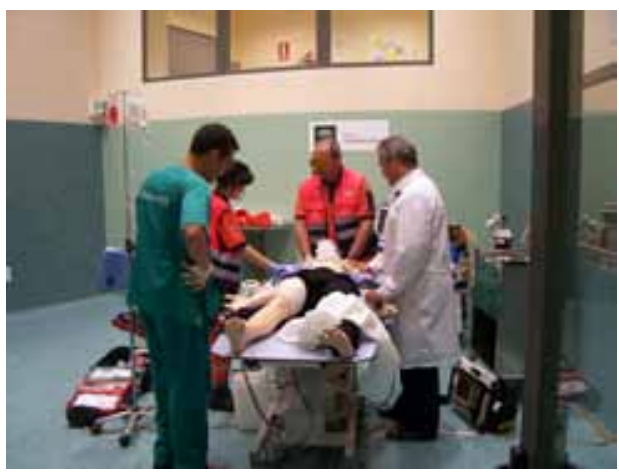

Figura 2. Sesión de trabajo de simulación robótica en la que se lleva a cabo la transferencia de un paciente traumático grave asistido por un equipo de emergencias prehospitalarias. El resto de los alumnos observan la simulación desde un aula. Imagen cedida por Fundación IAVANTE (Chaves, J).

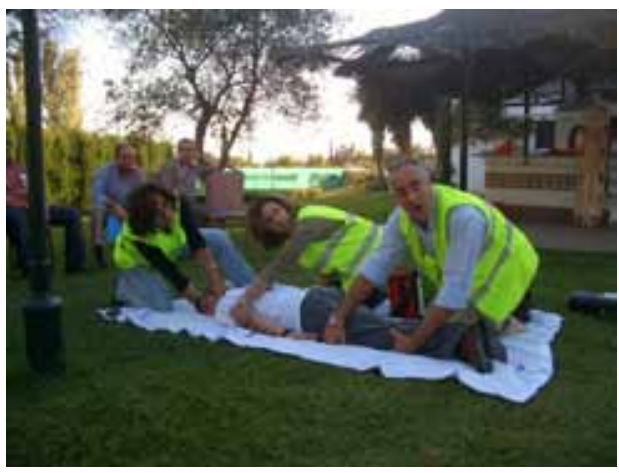

Figura 3. Profesionales de urgencias mostrando sus destrezas ante una situación simulada donde han encontrado un paciente precipitado con lesiones traumáticas graves.

la asistencia a estas patologías complejas. Después de la simulación, se realiza una sesión de revisión y debate de lo que ha ocurrido, con el apoyo del análisis en vídeo. 
Con ello, es posible poner al descubierto las deficiencias en la asistencia, ya sean debidas a errores técnicos o de evaluación del paciente, o bien derivadas de errores o carencias en habilidades no técnicas como el trabajo en equipo o el liderazgo ${ }^{2,3,23,24}$. En estos cursos, el trabajo previo de los do- centes es decisivo, ya que se requiere el diseño riguroso de los casos que se van a proponer (Tabla 3). En todo caso, se requiere que el equipo docente tenga un profundo conocimiento de las herramientas didácticas de simulación ${ }^{2,3}$.

Tabla 3. Diferentes situaciones que pueden programarse con simuladores avanzados.

\begin{tabular}{|l|l|}
\hline Vía aérea & $\begin{array}{l}\text { Laringoespasmo, hematoma retrofaríngeo, edema de lengua, trismus, respuesta verbal y } \\
\text { rigidez de nuca. }\end{array}$ \\
\hline Ventilación & $\begin{array}{l}\text { Movimientos respiratorios y sonidos respiratorios, así como disminución de la compliance } \\
\text { pulmonar }\end{array}$ \\
\hline Circulación & $\begin{array}{l}\text { Tonos cardiacos normales y anormales, así como pulso carotídeo bilateral, femoral bilateral, } \\
\text { braquial y radial en brazo izquierdo. }\end{array}$ \\
\hline
\end{tabular}

Modificado de Chaves Vinagre J y Villén Sánchez JA. Formación en trauma grave. En Ayuso Baptista F y col. Manejo integral de pacientes traumatizados graves. Ed. ARAN. (En prensa).

\section{BIBLIOGRAFÍA}

1. Grupo de trabajo «Proceso Asistencial Trauma Grave" de EPES. Informe inicial (Documento cero). Empresa Pública de Emergencias Sanitarias. Consejería de Salud. Junta de Andalucía. Abril 2008.

2. Chaves Vinagre J, Villén SÁnchez JA. Formación en trauma grave. En: Ayuso Baptista F, Castro Jiménez RA, Fonseca del Pozo FJ, Jiménez Moral G, Sánchez Ortega R. Manejo integral de pacientes traumatizados graves. Ed. ARAN. 2010. (En prensa).

3. Chaves J. Simulación y entornos de aprendizaje: entrenamiento de competencias clínicas y relacionales. Monografías IAVANTE 2004.

4. Coll-Vinent B, Sánchez M, Nogué R, Miró O. La enseñanza de la medicina de urgencias y emergencias en las facultades de medicina: situación actual. Emergencias 2010; 22: 21-27.

5. Nogué R. Situación de la medicina de urgencias en las universidades españolas. Sociedad Española de Medicina de Urgencias y Emergencias. Secretaría de Formación. Disponible en: http:// www.semes.org/index.php?option=com_cont ent\&task=view\&id=38\&Itemid=101 gonsultada por última vez en mayo de 2009.

6. Hobgood C, Anantharaman V, Bandiera G, CameRON P, HaLPerin P, Holliman J et al. Federación Internacional de Medicina de Urgencias: Modelo de plan de estudios para la formación en Medicina de Urgencias y Emergencias. Emergencias 2009; 21: 451-455.

7. Directiva 2005/36/CE del Parlamento europeo y del Consejo, 7 de septiembre de 2005, relativa a reconocimiento de cualificación profesional.

8. European observatory on health care system: health care systems in transition, United Kingdom, 2003.

9. Winter and emergency service team, emergency care report, Departament of Health, 2002-2003.

10. Chodosas M. Etude de l'organisation des different systems d'urgences dans cinq pays europeens. Tesis doctoral. Universite Paris VII. Faculte de Medecine Lariboisiere-SaintLouis. 2003.

11. MoвсKе H. Emergency medicine in Germany. Ann Emerg Med 1998; 31: 111-115.

12. Langhelle A, Morten H, Silfvast T, Björnsson HM, LIPPERT F, ERSSON A et al. Emergency medical service system in the nordic countries. Scand J Trauma Resusc Emerg Med 2004: 12: 212-228.

13. Ley $44 / 2003$ de 21 noviembre de ordenación de las profesiones sanitarias (LOPS). BOE $\mathrm{n}^{\circ} 208$ de 22 de noviembre de 2003, p. 41442-41458.

14. Grupo de trabajo de la EuSEM. Plan europeo de estudios de Medicina de Urgencias y Emergencias. Emergencias 2009; 21: 456-470. 
15. Sociedad Española de Medicina de Urgencias y de Emergencias. Proyecto de programa docente de de la Especialidad de Medicina de Urgencias y Emergencias. 2008.

16. Proyecto de programa formativo troncal de las especialidades de medicina de urgencias y emergencias, medicina familiar y comunitaria y medicina interna. SEMES-SEMERGEN. 2008.

17. FRCPC. Residency in Emergency Medicine, Calgary, Health Region, university of Manitoba. Programa docente.

18. Montero Pérez FJ, Gosálbez Pequeño B, Muñoz Ávila JA. Cursos de formación en Medicina de Urgencias para médicos residentes: ¿sabemos de dónde partimos? Emergencias 1999; 11: 96-101.

19. Juárez Alonso S. La enseñanza de la Medicina de Urgencias, una asignatura pendiente. Emergencias 1999; 11: 91-92.

20. Real decreto $1125 / 2003$ por el que se establece el sistema europeo de créditos y el sistema de calificaciones en las titulaciones universitarias de carácter oficial y de validez en todo el territorio nacional. BOE $224,18 \mathrm{de}$ setiembre de 2003, p. 34355-34356.

21. González Armengol JJ, Carricondo F, Mingorance C, Gil-Loyzaga P. Telemedicina aplicada a la atención sanitaria urgente: aspectos metodológicos y prácticos. Emergencias 2009; 21 : 287-294.

22. González Gómez JM, Chaves Vinagre J, Ocete Hita E, Calvo Macías C, Grupo Docente de Simulación Pediátrica de la Fundación IAVANTE. Nuevas metodologías en el entrenamiento de emergencias pediátricas: Simulación médica aplicada a pediatría. An Pediatr (Barc) 2008; 68: 612-620.

23. Block EF, Lottenberg L, Flint L, JAKobSEn J, LieBNITZKY D. Use of a human patient simulator for the advanced trauma life support course. Am Surg 2002; 68: 648-651.

24. Luis Navarro JC, Sánchez Peña J, Gutiérrez CresPO A. Simuladores anestésicos en la docencia. Cap 42. En: Carrasco Jiménez MS y col. Fundamentos de Anestesiología. Medicina Crítica y Tratamiento del dolor, vol II, Ed Grupo Editorial Universitario, Granada 2003. 
